\title{
Integral Analysis of the Process of Electro-Remediation of Andisols Polluted by Heavy Metals
}

\author{
M.V. Vázquez,,${ }^{a,{ }^{*}}$ F. Hernández-Luis, ${ }^{b}$ M. Lemus, ${ }^{\text {b C.D. Arbelo }}{ }^{c}$ \\ a) Instituto de Química (Electrochemical Group), Faculty of Exact and Natural Sciences, \\ Universidad de Antioquia, A.A.1226, Medellin, Colombia \\ ${ }^{b)}$ Department of Physical Chemistry, Universidad de la Laguna, Tenerife, España \\ ${ }^{c}$ Department of Soil Science and Geology, Universidad de La Laguna, Tenerife, España
}

Received 25 March 2004; accepted 15 June 2004

\begin{abstract}
An integral analysis was carried out on the results obtained in laboratory experiments on electro-remediation of soils using two heavy metal-contaminated volcanic soils from the region of Antioquia, Colombia. Measurements of $\mathrm{pH}$, conductivity, and pollutant concentration were made on each soil. The results were related to the structural characteristics of the soils employed in the experiments. Under the present experimental conditions it was shown that the soil with the lower concentration of organic matter had a lower $\mathrm{pH}$ regulatory capacity, that treatment without using wash solutions was more efficient for the mobilization of the metals, and that the particular characteristics of each soil conditioned the efficiency of the treatment.
\end{abstract}

Keywords: andisols, soil pollution, electro-remediation, heavy metals, regulatory capacity.

\section{Introducción}

El suelo es un cuerpo natural formado por una conexión de elementos y procesos, resultado de su localización y del contacto de la atmósfera con la superficie de la corteza. Esta delgada piel de la superficie responsable de la existencia de la vida en el planeta es, por lo tanto, un medio de elevada complejidad debido a que se trata de la interacción de distintas fases y materiales: compuestos inorgánicos, materia orgánica, aire, agua, micro-organismos, elementos nutritivos, etc. 
Esta misma complejidad motiva que cualquier método empleado para el análisis de suelos debe implicar necesariamente la integración de varios resultados experimentales.

La importancia de un correcto análisis de los suelos radica en que con esta caracterización fisicoquímica del material se tiene información acerca de la fertilización, especialmente en aquellos cultivos anuales cuyas raíces exploran una capa uniforme de suelos.

Existen distintos procedimientos experimentales de rutina para el análisis de suelos y pueden tener distintos objetivos. Así, si se desea analizar la fertilidad del material será importante analizar parámetros tales como: $\mathrm{pH}$, textura, materia orgánica, contenido de fósforo, de potasio, etc.

Por otra parte, si el interés está centrado en la caracterización del suelo será necesario determinar: contenido en materia orgánica, de fósforo, potasio, aluminio, $\mathrm{pH}$, capacidad de intercambio catiónico, textura, contenido de calcio, magnesio, sodio, etc.

Por último para interpretar la salinidad que presenta el suelo se analizará el porcentaje de carbono, de aluminio, $\mathrm{pH}$, contenido de carbonato de calcio, humedad, capacidad de intercambio catiónico, bases totales, porcentaje de saturación de bases y salinidad.

Esta variedad de procedimientos se aplicarán considerando como material de partida suelos en estado natural pero, si el interés se orienta al análisis de zonas que pudieran presentar contaminación, por ejemplo de metales, será necesario ampliar este listado de determinaciones. En este sentido se debe tener en cuenta que tanto fenómenos naturales como otros derivados de actividad humana, uso de fertilizantes, pesticidas, derrame de residuos contaminados, actividades industriales, etc., serán potenciales fuentes de contaminación de suelos [1].

Dentro de las posibilidades experimentales que ofrece la electroquímica, existen herramientas que pueden colaborar en el entendimiento del comportamiento de suelos de distintas características sometidos a procesos de contaminación y posterior tratamiento. En este sentido es posible emplear dichas técnicas para el análisis, monitoreo y tratamiento de suelos contaminados. 
Una de estas posibilidades lo constituye la electro-remediación de suelos. Esta técnica implica la aplicación de un campo eléctrico continuo entre dos electrodos enterrados en el suelo, durante un cierto tiempo. Con la aplicación de este campo eléctrico se consigue, dependiendo de diversos factores como el grado de humedad del terreno, acidez, etc., movilizar las especies cargadas hacia los electrodos correspondientes (ánodo o cátodo) [2-12]. La electro-remediación permite, en comparación con otras técnicas de limpieza, una menor exposición de los operadores a los contaminantes, realizando una acumulación de los mismos en las cercanías de los electrodos, permitiendo de esta forma una posterior disposición de los residuos así concentrados.

Un factor importante que debe ser tenido en cuenta en los tratamientos de este tipo, es la electrólisis del solvente que ocurre en los electrodos y que genera la aparición de frentes ácidos y básicos. Estos frentes al movilizarse por el interior del suelo, pueden interferir de modo apreciable en la eficacia de la remoción de los contaminantes: por ejemplo el precipitar los metales como hidróxidos.

Es importante destacar que si bien la técnica de electro-remediación implica un tratamiento relativamente largo (varias semanas dependiendo del grado y tipo de contaminación), es posible emplear los fundamentos de la misma en estudios de laboratorio que permitan caracterizar distintos materiales.

En el presente trabajo se empleó como material de estudio muestras de andisoles de la región de Antioquia (Colombia). Este tipo de suelos se caracteriza por su origen volcánico y se encuentra en zonas andinas donde se realizan importantes actividades agrícolas.

En este artículo se presentan los resultados obtenidos al aplicar el tratamiento de electro-remediación en laboratorio, a dos andisoles con distintas características (distinto origen) contaminados con iones plomo y cadmio.

\section{Materiales y métodos}

\section{Celda de electro-remediación}

Como celda de electro-remediación se empleó un diseño construido en nuestro laboratorio, que permite incorporar una mezcla de 200 gramos de suelo con 100 
$\mathrm{mL}$ de solución contaminante o agua desionizada, según el caso. En la Fig. 1 se presenta un esquema de la celda indicando las principales características de la misma.

En los experimentos donde se empleo un flujo de agua para disminuir el efecto de la creación de frentes ácidos y alcalinos, se hizo circular por cada cámara agua desionizada a una velocidad de $1 \mathrm{~mL} / \mathrm{min}$, aproximadamente.

Un campo eléctrico de $175 \mathrm{~V} . \mathrm{m}^{-1}$ fue establecido entre dos electrodos de grafito de $2.15 \mathrm{~cm}^{2}$ de área durante 8 y 48 horas, respectivamente, aplicando una diferencia de potencial continuo de $35 \mathrm{~V}$, generado por una fuente HEALT.

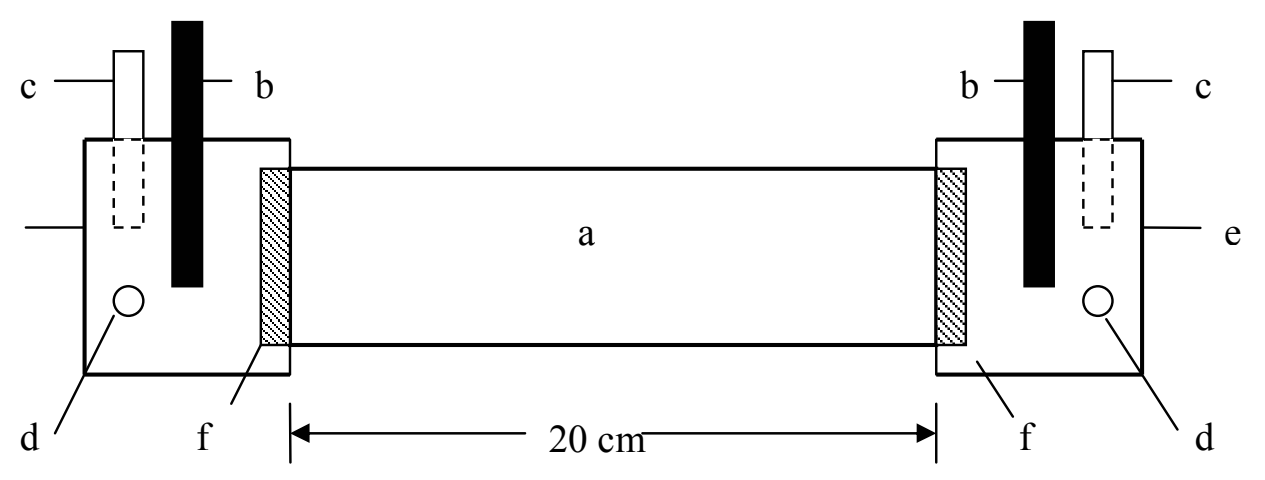

Figura 1. Diagrama esquemático de la celda de electro-remediación utilizada. a - suelo contaminado; b - electrodos de grafito; c - entrada solución de lavado; d - salida solución de trabajo; e - ventana transparente: f - rejilla perforada.

\section{Suelo estudiado}

En el presente trabajo se utilizaron dos suelos con distintas características estructurales: uno proveniente del oriente antioqueño, denominado Suelo $O \mathrm{y}$ otro de la región del Urabá antioqueño que fue designado como Suelo I. En la Tabla 1 se presentan las principales características de cada uno de los suelos estudiados.

Como contaminante se empleó una solución $0.1 \mathrm{M}$ de $\mathrm{Pb}\left(\mathrm{NO}_{3}\right)_{2}$ y $\mathrm{Cd}\left(\mathrm{NO}_{3}\right)_{2}$ de grado analítico, preparadas con agua desionizada. 
Tabla 1. Características de los suelos estudiados.

\begin{tabular}{lcc}
\hline & Suelo O & Suelo I \\
\hline Arena / \% & 40 & 70 \\
Limo / \% & 40 & 16 \\
Arcilla / \% & 20 & 14 \\
$\mathrm{pH}$ & 5.2 & 5.5 \\
Materia orgánica / \% & 18.1 & 1.0 \\
Capacidad de intercambio efectiva / meq $100 \mathrm{~g}^{-1}$ & 16.3 & 16.4 \\
\hline
\end{tabular}

\section{Procedimiento}

En la Fig. 2 se resume el procedimiento seguido en los experimentos de electroremediación presentados en este trabajo.

\section{Instrumental}

El contenido de los metales pesados residuales después del tratamiento fue determinado polarográficamente en un equipo BAS CV50W empleando la técnica OSWV.

Las medidas de conductividad se llevaron acabo con un equipo SCHOTT Handdylab LF1, directamente en las suspensiones antes de la etapa de filtración para el posterior análisis químico.

El pH se midió utilizando un pHmetro ORION SA720.

\section{Resultados}

\section{pH del suelo después del tratamiento de electro-remediación}

En la Fig. 3 se muestran los resultados obtenidos al analizar los distintos fragmentos de los suelos estudiados, una vez finalizado el tratamiento de electrólisis. Para comparación se incluye en las gráficas el valor de $\mathrm{pH}$ correspondiente a las muestras tomadas como blanco del experimento, es decir suelos contaminados sin someterlos a campo eléctrico. De acuerdo a las reacciones de formación de oxígeno e hidrógeno en el ánodo y cátodo, respectivamente, se espera que los segmentos más próximos a las cámaras de los electrodos se vean afectados por los frentes ácido y alcalino que ahí se generan. En este sentido, se observa que el Suelo I presenta una menor capacidad 
reguladora del $\mathrm{pH}$ especialmente cuando el tratamiento se realiza sin el paso de solución de lavado por las cámaras.

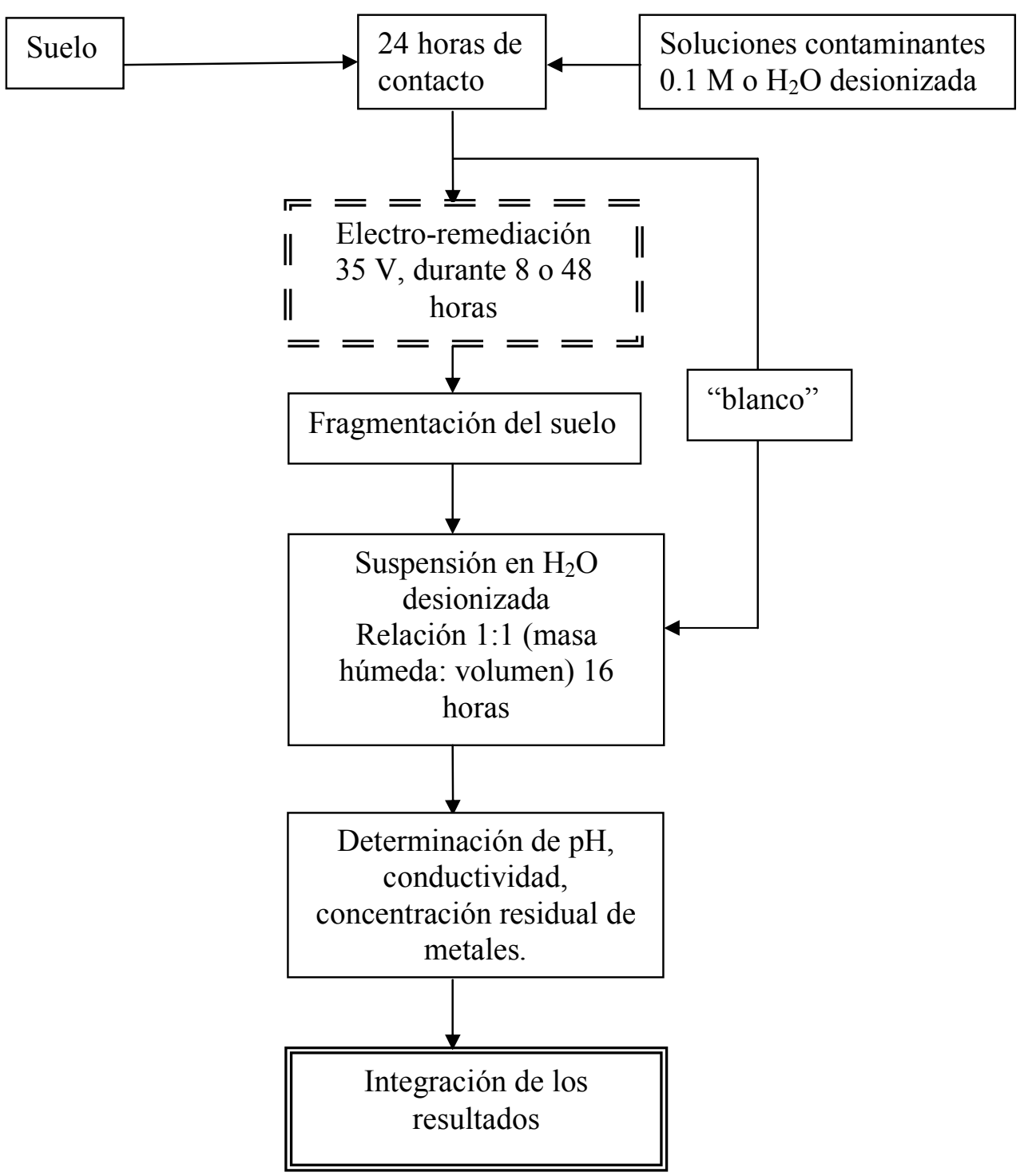

Figura 2. Diagrama del procedimiento empleado en los experimentos.

\section{Variación de la conductividad residual}

Cuando se mide la conductividad que presentan las suspensiones de los distintos fragmentos de suelo, después del tratamiento de electro-remediación, se obtienen los resultados mostrados en la Fig. 4. Nuevamente, se ha incluido el valor de 
conductividad que presenta cada suelo contaminado cuando no es sometido a tratamiento alguno. En este caso se verifica que en el Suelo $O$ se insinúa un movimiento neto de iones hacia los extremos de la celda, especialmente cuando se utilizan soluciones de lavado. En ambos suelos se presenta el mismo comportamiento en el segmento más próximo a la cámara del cátodo, es decir el menor valor de conductividad. Esto es indicativo de una menor presencia de especies cargadas en esta zona del suelo contaminado. Puede considerarse una evidencia indirecta de la formación de especies insolubles (hidróxidos) debido al valor alto de $\mathrm{pH}$ que se genera en esta zona, como se muestra en la Fig. 3.

\section{Variación del contenido de metal residual luego del tratamiento de remediación}

En la Fig. 5 se muestran los valores de concentración, expresados como valores relativos a la cantidad de metal presente en los suelos tomados como blanco (suelos sin electro-remediar).

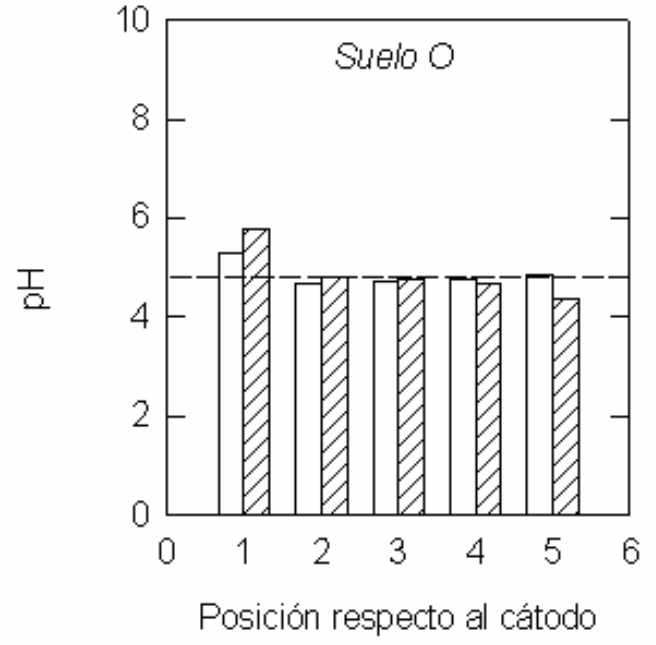

con lavado

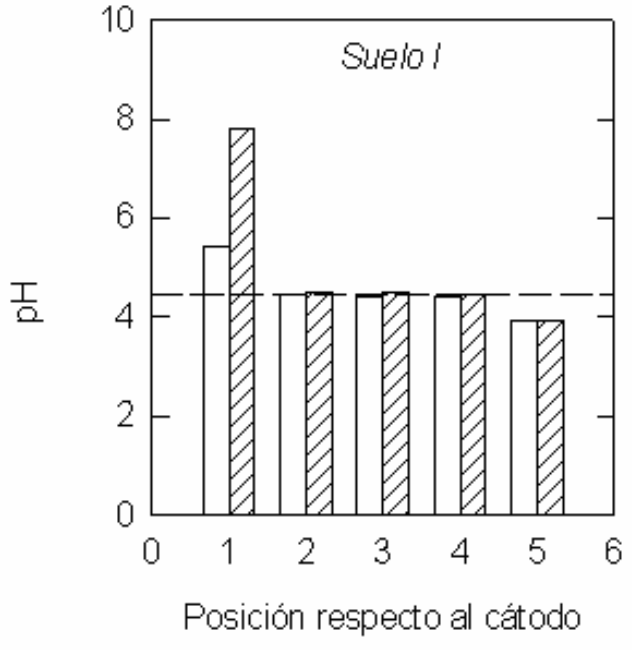

QZT sin lavado
---- "blanco"

Figura 3. Variación del pH en función de la posición respecto al cátodo para cada uno de los suelos estudiados.

Del análisis de las variaciones observadas puede afirmarse que los procesos electrocinéticos que permiten la movilización de los metales resultan más efectivos cuando no se emplea flujo de solución de lavado por las cámaras de los 
electrodos. Esto se cumple especialmente para el Suelo O. El aumento de metal libre con relación al blanco que se observa, especialmente para el plomo y cuando se aplica solución de lavado, podría explicarse teniendo en cuenta la disminución de $\mathrm{pH}$ que se produce a raíz del tratamiento de electro-remediación. Esta acidificación local influirá en gran medida en los equilibrios de adsorción que se presentan entre los metales y las partículas de suelo.
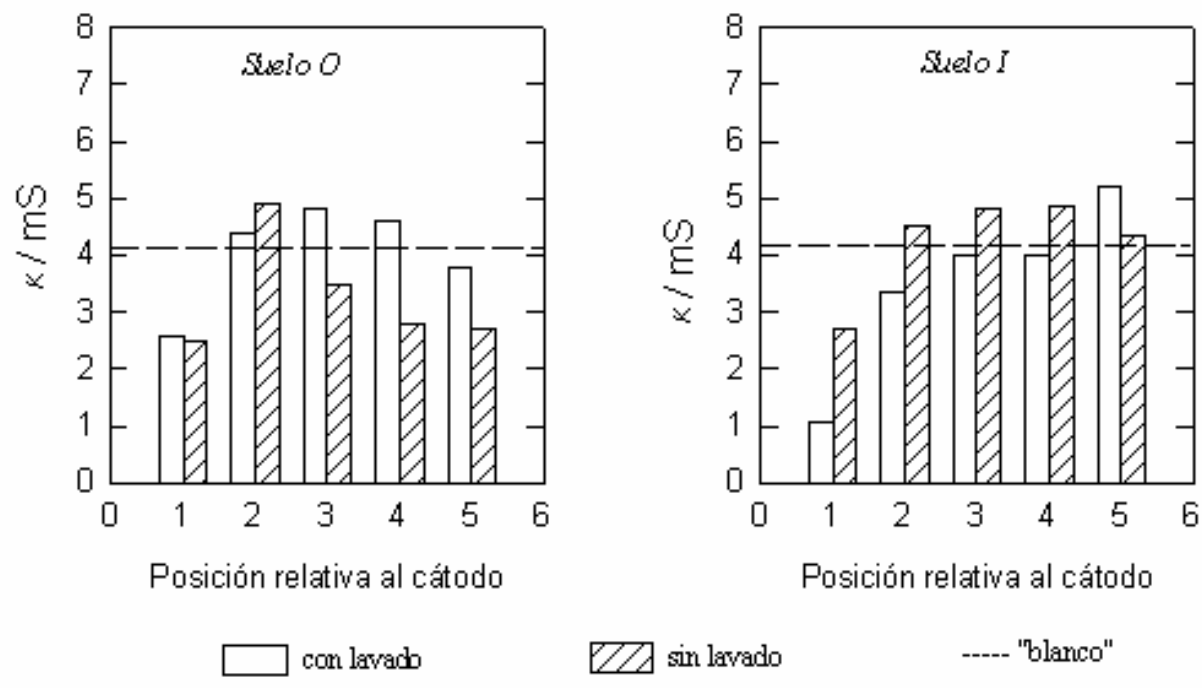

Figura 4. Variación de la conductividad en función de la posición respecto al cátodo para cada uno de los suelos estudiados.

\section{Discusión}

Analizando el comportamiento mostrado por los dos suelos bajo estudio, al ser sometidos a un campo eléctrico durante un tiempo relativamente corto de tratamiento, se puede afirmar que existe una estrecha relación entre los tres parámetros estudiados: $\mathrm{pH}$, conductividad y concentración de metal. De esta manera, por ejemplo, se aprecia que el Suelo $O$, de mayor contenido de materia orgánica es el que muestra mayor capacidad reguladora de $\mathrm{pH}$ y por lo tanto en el que se presentará menor formación de especies insolubles, lo que a su vez se refleja en un valor no tan pequeño de conductividad eléctrica. Por otra parte, con relación a la efectividad del tratamiento para movilizar iones, se puede afirmar que ésta es mayor cuando no son lavadas continuamente las cámaras de los 
electrodos. Esto implica que existe un mayor "tiempo de contacto" entre el suelo y la solución electrolítica que se va generando en las respectivas cámaras a medida que el tratamiento de remediación se lleva a cabo. Esta conclusión es de importancia, especialmente de cara al diseño de un tratamiento en campo, puesto que se simplificaría el mismo y se disminuiría el costo del tratamiento al no emplear el flujo de agua (que requeriría en ese caso un posterior tratamiento de limpieza).
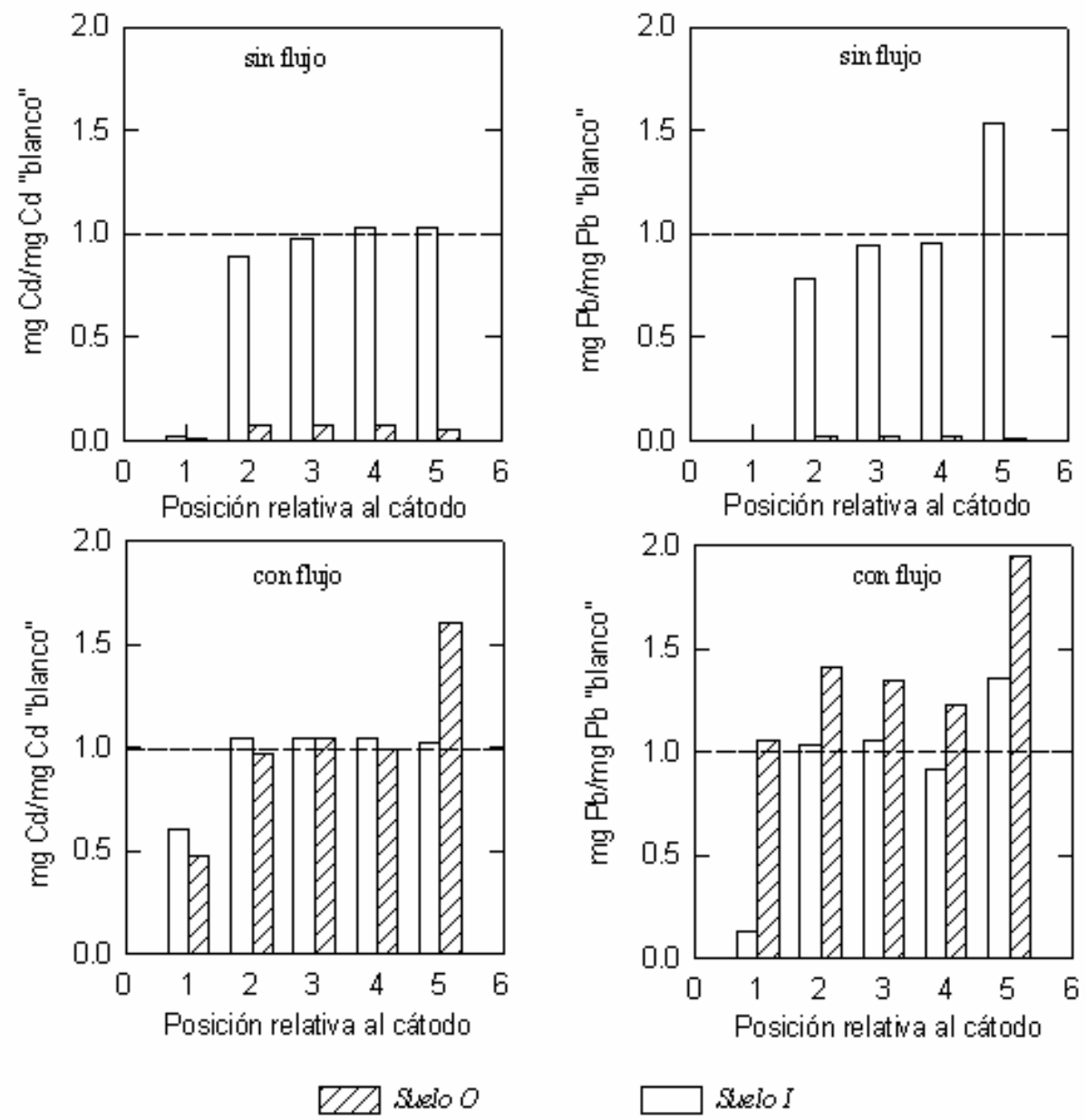

Figura 5. Concentración residual de cadmio e plomo después de la electro-remediación.

Las medidas de conductividad no sólo reflejan la formación de especies insolubles, sino que también permiten vislumbrar que existe un movimiento iónico causado por el mecanismo de difusión. Es decir, se produce movimiento hacia el exterior del suelo y por lo tanto se evidencia con mayor claridad en los 
segmentos más próximos a las cámaras de los electrodos. Este mecanismo de transporte se acentúa si se aplica solución de lavado, al aumentar el gradiente de concentración.

A modo de comparación, y para analizar si los efectos señalados obedecen a la presencia de los metales pesados o son inherentes al tipo de suelo en tratamiento, se realizó un estudio similar, pero con mayor tiempo de remediación (48 horas), y empleando una celda totalmente transparente para verificar los movimientos de suelo que se producen durante la electro-remediación. En la Fig. 6 se muestran los resultados obtenidos para los dos suelos estudiados.

Analizando la variación de $\mathrm{pH}$ se comprueba que el comportamiento señalado antes está relacionado con el tipo de suelo y no con el contaminante utilizado en este caso, ya que nuevamente el Suelo $I$ es el que presenta menor capacidad reguladora de $\mathrm{pH}$. Se debe tener en cuenta que, aún cuando no se agreguen compuestos iónicos, con la carga natural que presentan los suelos se producen las reacciones de electrólisis en las cámaras de los electrodos, lo que provoca que al finalizar el experimento se tengan valores para la cámara del cátodo de $11.09 \mathrm{y}$ 11.59 y para el ánodo de 2.32 y 2.16 para los Suelos $O$ e $I$, respectivamente.

Con relación a la variación de la conductividad se puede considerar que ésta obedece, por una parte a la precipitación causada por el aumento local de $\mathrm{pH}$ en los segmentos más próximo al cátodo y por otra al movimiento de partículas de suelo hacia la cámara del ánodo, lo que es visible a simple vista como un aumento gradual en turbidez en la mencionada cámara.

Puede concluirse entonces que los factores que influyen en mayor medida en la eficiencia de movilización están relacionados principalmente con las características de los suelos en cuestión. Por una parte, del contenido de arcilla (20\% del Suelo $O$ frente al $14 \%$ del Suelo I) y por otra y con mayor influencia del contenido de materia orgánica (18.1\% para el Suelo $O$ frente a un $1 \%$ del Suelo I). 

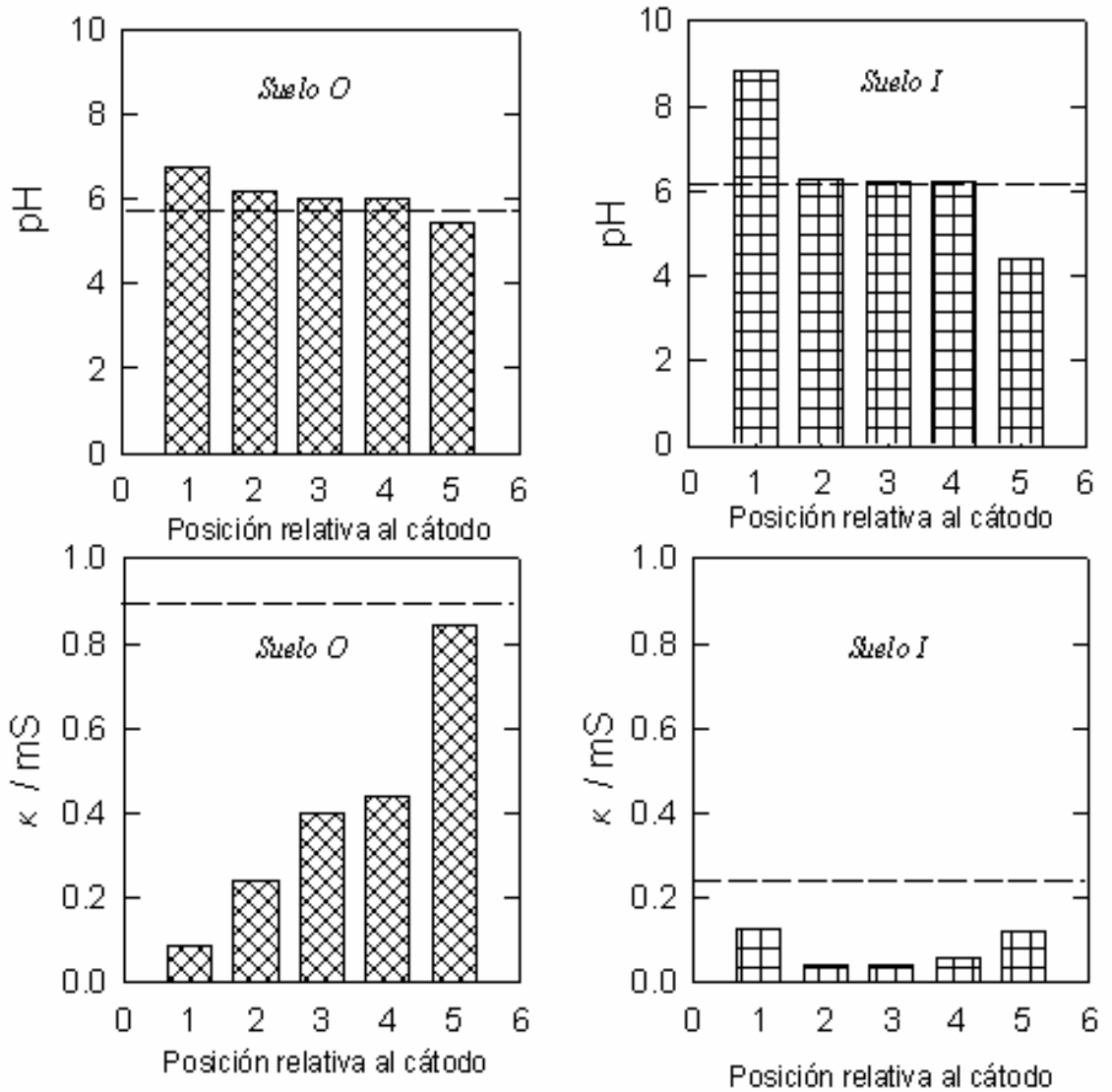

Figura 6. Valores del $\mathrm{pH}$ y de la conductividad en función de la posición al cátodo para los suelos estudiados sin plomo ni cadmio añadidos y sin flujo de lavado.

De esta manera se pone de manifiesto la utilidad de este tipo de experimentos de electro-remediación en laboratorio ya que, realizando tratamientos relativamente cortos en tiempo y realizando un posterior análisis integral, se pueden relacionar todo lo observado con características propias de los suelos.

\section{Agradecimientos}

Los autores agradecen a la Universidad de La Laguna (Proyecto Grupos Precompetitivo 1802440302) y a la Dirección General de Universidades del Gobierno de Canarias (Proyecto PI2003/138) por financiar parte de esta investigación. 


\section{Análisis Integral del Proceso de Electro-Remediación de Andisoles Contaminados con Metales Pesados}

\section{Resumen}

Se realizó un análisis integral de los resultados obtenidos en experimentos de electroremediación en laboratorio para dos suelos volcánicos de la región de Antioquia (Colombia), contaminados con metales pesados.

Para los estudios se analizaron conjuntamente medidas de $\mathrm{pH}$, conductividad y concentración de contaminantes. Los resultados fueron relacionados con las características estructurales de los suelos empleados en los experimentos.

Se comprobó que, para las condiciones experimentales empleadas, el suelo con menor contenido de materia orgánica, presenta una menor capacidad reguladora de $\mathrm{pH}$, que el tratamiento sin emplear soluciones de lavado presenta mayor eficiencia para la movilización de los metales y que las características de los suelos son las que condicionan la eficacia del tratamiento.

Palabras clave: andisoles, contaminación de suelos, electro-remediación, metales pesados, capacidad reguladora.

\section{References}

1. H.E. Allen, C.P. Huang, G.W. Bailey, A.R. Bowers, "Metal Speciation and Contamination of Soil", Lewis Publishers, CRC Press, 1995.

2. M.V. Vázquez, D.A. Tobón, Afinidad 58 (2001) 437.

3 M.M. Page, C.L. Page, J. Environm. Eng. (2002) 208.

4. M.V. Vázquez, D.A. Tobón, F.L. Gutiérrez, Afinidad 59 (2002) 601-604.

5. M.V. Vázquez, F. Hernández-Luis, Innovación 15 (2003) 45.

6. S. Chinthamreddy, K. Reddy, J. Soil Contamination 8 (1999) 197.

7. S. Pamukcu, A. Weeks, J. Wittle, J. Hazardous Mater. 55 (1997) 305.

8. $\quad$ K. Reddy, S. Chinthamreddy, Waste Management 19 (1999) 269.

9. K. Reddy, S. Chinthamreddy, A. Al-Hamdan, Remediation (2001) 85.

10. K. Reddy, M. Donahue, R. Saichek, R. Sasaoca, J. Air Waste Manag. Assoc. 99 (1999) 823.

11. K. Reddy, U. Parupudi, S. Devulapalli, C. Xu, J. Hazardous Mater. 55 (1997) 135.

12 K. Reddy, A.B. Shirani, Geotech. Geolog. Eng. 15 (1997) 3. 